\title{
被 \\ LA INTENCIÓN EDITORIAL DE CALDERÓN Y LA EDICIÓN DE LAS COMEDIAS DE LA CUARTA PARTE
}

\author{
Juan Luis Suárez y Graciela Manjarrez \\ The University of Western Ontario
}

[Anuario calderoniano (ISSN: 1888-8046), 1, 2008, pp. 317-332]

Dos cosas parecen tener en común las comedias de la Cuarta parte de Calderón: de un lado, la construcción argumental mayoritaria en función de técnicas «mitologizantes» ${ }^{1}$ - ya sea la utilización temática de fábulas mitológicas como en el caso de Eco y Narciso o El golfo de las sirenas, ya sea la «mitologización» de personajes o asuntos históricos y temas legendarios que se lleva a cabo en otras como La aurora en Copacabana o El gran príncipe de Fez-; de otro, lo que parece conectar a todas las obras es el proceso de «socialización» al que se ven sometidas estas doce comedias al convertirse en productos de la imprenta.

Fue Alan K. G. Paterson quien llamó la atención sobre la condición social de los textos dramáticos al retomar una idea que Jerome J. McGann había expuesto en su libro de 1991, The Textual Condition. Para Paterson, la condición textual de las comedias de Calderón se ve sustancialmente afectada por la diferente socialización ${ }^{2}$ que sufren en cuanto género leído y, por tanto, sujeto a los avatares de la imprenta,

${ }^{1}$ Suárez, 2002.

2 «Y en efecto, interrogándolo desde este ángulo, el paratexto ofrece un paralelismo sugestivo entre la incorporación del texto de las comedias en su forma li- 
respecto del tipo de socialización que había determinado su trayectoria inicial en cuanto textos preparados por el autor para la vida escénica. «La condición textual enunciada por McGann — dice Patersonesquiva la idealización del texto literario. En su evolución el texto está sujeto a la intervención de los que lo usan y manejan, entre quienes figura por supuesto el mismo escritor. Las manifestaciones corpóreas del texto son testimonios de su progresiva socialización» $»^{3}$. Estas manifestaciones corpóreas del texto ofrecen, en determinados casos, una clave de entrada a los textos, la cual queda garantizada por la exitosa combinación de las mejores técnicas de la crítica textual con las de la hermenéutica. De esta forma, Paterson se detiene, especialmente, en el paratexto - «ese territorio que linda con el mundo de la ficción sin serlo» ${ }^{4}$ - de la Primera parte de comedias recogidas por Joseph Calderón (1636), para intentar determinar así las razones que mueven a Calderón a entrar en el mundo de la edición. Según Paterson, el título de esta edición recoge la intención de

seguir publicando las comedias de Calderón en una serie que empieza precisamente con esta Primera parte. Retrospectivamente, el título hace tabla rasa de cualquier otra publicación no autorizada. La promesa de futuras partes que es implícita en el título se cumplió efectivamente hasta que la piratería intervino simulando una Quinta parte que cortó el hilo que luego iba a recoger Juan de Vera Tassis. No vacilo en concluir que la intencionalidad a que me refiero es autorial; el mismo Calderón inicia y da noticia del trasvase de su teatro al libro ${ }^{5}$.

Si en los preliminares de la Primera parte lo que encontramos es una socialización que se manifiesta al desvelarse el paralelismo, según Paterson, entre la fijación del texto calderoniano a través de la estabilidad del libro y su ascenso social al mundo caballeresco, los de la Cuarta parte ofrecen un panorama distinto, en el que destacan «la in-

bresca y la elevación de su creador a caballero de hábito. Por una parte, el paratexto, incluso y sobre todo en aquellas zonas reservadas para los protocolos administrativos del libro (aprobaciones, licencias, tasa, fe de erratas), sujeta las comedias al libro y a la imprenta; quiero decir que los preliminares asisten a la transformación de la comedia de género representado en género leído», Paterson, 2001, p. 21.

${ }^{3}$ Paterson, 2001, p. 19.

${ }^{4}$ Paterson, 2001, p. 19.

5 Paterson, 2001, pp. 19-20. 
quietud ocasionada por el mismo libro...», «el desorden que provocan los múltiples agentes que gestionan la proliferación de textos...» y el afán casi obsesivo del autor de "controlar su propia textualidad» ${ }^{6}$. En todo este proceso de socialización la inestabilidad que caracteriza al mundo editorial - el mismo dramaturgo es un agente entre muchos otros- se va imponiendo como la característica más sobresaliente y las notas pergeñadas por Calderón en los preliminares de comedias y autos van dejando a la vista la estrategia editorial que el mismo Calderón diseñó. Dice Paterson:

Ésta es la conclusión principal a la que llego: el futuro que los dos editores aseguran para los textos de Calderón es condicionado por el pasado que él mismo fraguó en la prolongada lucha que mantuvo con la materia literaria que se resistía a plasmarse en formas ideales. Fue él quien creó a su medida el papel de editor, fiel a su empresa. Su empresa consistía en enaltecer y pulir su obra en su elevación al libro, garantizándole una preeminencia en las primeras filas de la literatura nacional. Las partes de Vera Tassis y sus sucesores son un testimonio duradero del éxito de la estrategia ideada por Calderón. Los tomos de Pando y Mier y sus sucesores ofrecen un caso muy parecido. Ambos influyen profundamente en la formación y percepción de un Calderón clásico y canónico ${ }^{7}$.

Así, nos encontramos ante la reacción del autor frente a esa inestabilidad que Don W. Cruickshank tipificó siguiendo los ejemplos que el propio Calderón había dado en la dedicatoria a la Cuarta parte «de casi todas las desventuras a que pueden verse sometidas las comedias impresas» ${ }^{8}$. A estos ejemplos, añade Cruickshank uno más que se deriva directamente de la estrategia calderoniana: «la intervención del editor» ${ }^{9}$. Es en esta figura del editor donde confluyen la inestabilidad del texto dramático en su vida por imprentas y librerías y los intentos del autor de fijar su texto por razones económicas y de prestigio. En gran medida, estamos asistiendo, pues, a la transformación del autor en editor de sus propias comedias. Una transformación a la que

${ }^{6}$ Paterson, 2001, pp. 22-23.

7 Paterson, 2001, pp. 28-29. Para todo lo relacionado con la Primera parte de las comedias de Calderón hay que tener presente a partir de ahora el trabajo de Luis Iglesias Feijoo y el grupo calderoniano de Santiago (2006).

${ }^{8}$ Cruickshank, 2000, p. 129.

9 Cruickshank, 2000, p. 129. 
ha de atender el editor moderno no sólo porque parece que esa fuera la intención editorial de Calderón, sino porque es en la tradición impresa, en lo que se refiere a la Cuarta parte, en donde encontramos las mejores pruebas textuales para la elaboración de esa edición filológica.

La transformación de Calderón autor en editor de sus propias obras la describe él mismo en el prólogo al amigo ausente de la Cuarta parte de 1672 (Josef Fernández de Buendía como impresor y Antonio de la Fuente como librero) que luego recogerán también las dos impresiones de 1674 (con Calatayud y de la Fuente como libreros respectivos). En él Calderón habla de los libros de comedias en que andan algunas de sus obras «esparcidas» ${ }^{10}$, hasta que la petición de su ficticio corresponsal le hace caer en la cuenta de lo que se va publicando con su nombre en esos libros. La «desimaginación» inicial se transforma entonces en un celo editorial que tiene en la enumeración de los tipos de errores y en la confección de la «anti-lista» de comedias sus más agudos síntomas y que se confirma con el recurso al amigo que le recomienda publicar sus propias comedias:

—Pues no tiene remedio lo pasado, enmendad lo porvenir. — ¿Cómo? -le pregunté.Y él me respondió: - Imprimiendo vos vuestras comedias atajaréis la sinrazón de que otro las imprima. — Si veis —le dije- que ya no las busco para enviarlas, sino para consumirlas, ¿̇cómo me aconsejáis el aumentarlas? A que me respondió: - Sabed que hay persona que de las últimas que aún no han corrido esa fortuna tiene para imprimir un libro y es tan atento que, para no daros pesar, se ha valido de mí para que solicite vuestra permisión. - No me habléis en ella —le dije- porque no he de darla. - Pues tened entendido - prosiguió- que no es sola la persona por quien os pido quien la tiene y que de no imprimirlas él en Madrid, donde con mi asistencia salgan menos erradas, será sin duda que otros las envíen a Zaragoza o a Sevilla, de donde vendrán sin poderlo vos remediarlo, como las demás, mal corregidas. Viendo yo que el que empezaba en ruego acababa en amenaza, y amenaza tan factible, dándome no sé si al partido o al despecho: - Haced vos lo que quisiéredes - le dije-, pero con condición, si se imprimiere, que ha de ser la de Lucanor alguna de ellas... ${ }^{11}$

${ }^{10}$ Calderón de la Barca, Cuarta parte (1672).

11 Calderón de la Barca, Cuarta parte, fols. 4-5. 
Ya fuera por partido o por despecho el caso es que Calderón da en este prólogo de 1672 el paso definitivo para convertirse en editor de su propia obra ya sin necesidad de interponer un editor como había sido el caso de su hermano, es decir, sin ocultar su voluntad editora. El resultado de su decisión no va a ser, sin embargo, mucho más satisfactorio del que intentaba evitar mediante su supervisión del volumen de la Cuarta parte, o mejor, lo será desde el punto de vista de las atribuciones de las comedias, ya que la lista que ofrece el volumen de 1672 será la misma que se repita en las dos impresiones de 1674 y en la de Vera Tassis de 1688. El problema continuará, sin embargo, en lo que a los yerros se refiere en la mayoría de las comedias de esta parte; en este caso, el visto bueno del autor a un volumen ya confeccionado, según dice él mismo, no va a evitar los múltiples errores de varios tipos que siguen apareciendo en estos textos y que le llevarán a una participación mucho más activa en la edición de 1674 .

El proceso de socialización del que habla Paterson se concreta, pues, en un hecho de suma importancia para los editores modernos de las comedias contenidas en esta Cuarta parte: la declaración de la voluntad editora de Calderón a cargo del propio autor. Si la socialización a la que la imprenta somete a los textos dramáticos provoca el desdoblamiento profesional del autor, ahora también editor, la voluntad editora de Calderón afecta definitivamente a su transmisión textual posterior, es decir, a la historia de las doce obras de la Cuarta parte. Quizás la prueba más clara del efecto creado por la reacción en cadena provocada por la socialización y la intervención de Calderón sea la que ofrece el análisis filológico de su transmisión textual, ya que, en principio, todas las comedias responden a un mismo y definido esquema de transmisión en su vertiente impresa a partir de 1672 .

Este esquema de transmisión se caracteriza por los siguientes elementos: en primer lugar, hay una clara divergencia entre las historias que nos cuenta el rastreo de sus fuentes manuscritas e impresas anteriores a 1672, en los casos en que éstas existan, y la más uniforme que nos cuentan las fuentes impresas a partir de 1672. La existencia de fuentes manuscritas nos habla de obras con una génesis individual, a veces problemática, anterior a la publicación de la Cuarta parte. El caso paradigmático quizás sea el de El conde Lucanor, del que se hace eco el propio Calderón en su prólogo. Según Graciela Manjarrez, se conserva un manuscrito de la comedia en la Biblioteca Nacional de Ma- 
drid, pero no es autógrafo, sino que parece ser una copia de compañía con numerosos pasajes tachados y anotaciones en los márgenes relativas a la representación ${ }^{12}$. Su historia impresa está marcada por el aviso de Calderón acerca de las diferencias entre la comedia que se le atribuye con ese nombre y la que realmente es suya. También existen tres copias manuscritas de El postrer duelo de España, dos de las cuales contienen fechas anteriores a la primera edición de la Cuarta parte, y además una de ellas incluye enmiendas e intervenciones del propio Calderón ${ }^{13}$. En otros casos, como apunta Ezra S. Engling en su edición de La aurora en Copacabana, simplemente no se han encontrado manuscritos y la historia textual de la comedia se remonta sólo hasta la Cuarta parte de $1672^{14}$. Respecto a las comedias impresas antes de 1672 existen testimonios de indudable valor, como ocurre con la impresión vienesa de 1671 de Fineza contra fineza ${ }^{15}$, derivada directamente con toda probabilidad de la versión enviada por Calderón para la representación en Viena, pero no por ello más cercana a la intención editorial del autor.

Por el contrario, la tradición impresa presupone la colección, no las comedias consideradas individualmente. Así, todas las comedias revisadas hasta ahora (El postrer duelo de España, Fineza contra fineza, La

12 Al respecto señala: «La comedia "auténtica" de El conde Lucanor se conserva en un único manuscrito, no autógrafo, propiedad de la Biblioteca Nacional de Madrid (Ms.16386). No está fechado, ni tampoco figuran otros datos exactos que ayuden a ubicarlo cronológicamente. Consta de 71 folios numerados, bien dispuestos y copiados por una sola mano [...] El manuscrito presenta enmiendas al texto y algunos pasajes están tachados, especialmente en la segunda y tercera jornadas. Probablemente, los fragmentos estén así para su posterior supresión en la puesta en escena. Asimismo, en los márgenes del texto se pueden leer una serie de indicaciones que aluden a la interpretación, como es el caso de: "rodilla...y todo se representa con horror", a la entrada y salida de personajes y al sonido de "cajas". Por estas especificidades, posiblemente se trate de una copia manuscrita que utilizó alguna compañía teatral», Manjarrez, 2007, p. 758.

13 Rossetti, 1979, p. 7.

14 Engling, 1994, p. 17.

15 Según Cecilia Brain: «Fineza fue inicialmente compuesta por Pedro Calderón de la Barca por orden del emperador Leopoldo I y la emperatriz Margarita de Austria para la celebración del cumpleaños de Mariana de Austria (1634-1696), reina de España, y madre de la emperatriz, que se llevó acabo el 22 de diciembre de 1671 en Viena, Austria. La primera versión impresa se publicó en Viena, Austria, en la imprenta de Mateo Cosmerovio» (Brain, edición de Fineza contra fineza, trabajo inédito). 
aurora en Copacabana, Eco y Narciso, El conde Lucanor, El golfo de las sirenas, Apolo y Climene) siguen la misma trayectoria a partir del núcleo de 1672-1674-1688, una trayectoria que no vuelve a distinguir entre obras particulares hasta los trabajos de editores modernos en las últimas décadas del siglo xx. Éste es el caso, por ejemplo, de Vitse y Montarnall para El conde Lucanor, de Nielsen para El golfo de las sirenas, o de Engling para La aurora en Copacabana, de Guy Rosetti para El postrer duelo de España, o de Charles V. Aubrun para Eco y Narciso.

En segundo lugar, este esquema de transmisión nos habla de la importancia que adquiere a partir de 1672 la gestión de la marca editorial «Calderón» tanto por las razones económicas que el mismo Calderón declara como por las relacionadas con el prestigio social a que hace referencia Paterson. Esta gestión está consignada en la voluntad de Calderón de recuperar los derechos sobre sus textos mediante un procedimiento que combina la autentificación personal del propio autor de los títulos de sus comedias - frente a la persona interpuesta de otras partes, su hermano Josef - con la garantía de calidad «filológica» de los propios textos. Mientras que la Cuarta parte cumple con creces ese primer objetivo de autentificación de los títulos en la mayoría de las comedias, no ocurre así en el caso de la quizás más problemática de la colección, El conde Lucanor, sobre la que el propio Calderón insiste en que sea una de las incluidas en la parte para demostrar que - dice el autor- «aun las mías no lo son, pues hallará el que tuviere curiosidad de cotejarla con la que anda en la Parte quince que a pocos versos míos prosigue con los de otros; si buenos o malos, remítome al cotejo» ${ }^{16}$.

Por otra parte, menos exitoso es el control de calidad de los textos calderonianos, ya que las correcciones que el autor hace a las versiones contenidas en 1672 sólo se consiguen a costa de la producción de un número inusitado de errores nuevos en 1674. De este problema habla Engling al enumerar los testimonios de La aurora en Copacabana:

The relationship between B [1672] and $\mathrm{H}$ [1674, de Calatayud] is significant. The second edition $(\mathrm{H})$ is replete with variant readings (some of them of apparently authoritative, if anonymous, inspiration) but it also

16 Calderón de la Barca, Cuarta parte, fol. 5. 
derives from B. [...]. Contact between $\mathrm{B}$ and $\mathrm{H}$ becomes even more significant when one discovers that, despite its corrections and claim to greater accuracy, the final product of $\mathrm{H}$ is a version which is in many ways inferior to the editio princeps ${ }^{17}$.

Según Engling, «[i]n addition to the editorial aberrations just mentioned, $\mathrm{H}$ produced over 100 entirely new errors, thus undermining his own revised edition» ${ }^{18}$. Lo que ocurre es que, según Cruickshank, parece que la participación de Calderón en la segunda edición de la Cuarta parte fue mucho mayor de la que tuvo en la primera, ya que debido a los errores aparecidos en 1672 decidió revisar y corregir la de $1674^{19}$.

Esto ha llevado a una primera disyuntiva entre los editores modernos que se refleja bien en la argumentación de Engling y que puede formularse de la siguiente manera: puesto que la edición de 1674 no es mejor filológicamente que la de 1672 (algo que hay que contrastar comedia por comedia puesto que la que edita Engling es, junto a Eco y Narciso y El monstruo de los jardines, según Everett Hesse, una de las que más cambios sufre en 1674)20, lo más acertado sería editar a partir de 1672, que es la editio princeps y, temporalmente, está más cerca del original de Calderón. Esta argumentación presenta, sin embargo, varios errores. En primer lugar, la caracterización como editio princeps no tiene aquí la relevancia que en otros casos, puesto que a tenor de lo que dice el propio Calderón en el prólogo de 1672, él no las ha seleccionado (excepto El conde Lucanor) ni corregido, sino que «chantajeado» por el intermediario de su editor, da su permiso y les pone prólogo. Pero no parece que pueda decirse que la de 1672 es la edición de Calderón. Además, hemos visto que a partir de esta fecha toma un papel más activo como editor de su propia obra en lo que respecta a esta Cuarta parte y decide corregir las comedias para la edición de 1674. Por otra parte, aunque la editio princeps hubiera sido suya, el haber cambiado de opinión debería ser suficiente para considerar más cercana a la voluntad del autor, a su voluntad editora, la de

17 Engling, 1994, p. 24.

18 Engling, 1994, p. 27.

19 Cruickshank, 1973, pp. 18-21.

${ }^{20}$ Hesse, 1948, p. 235. 
1674. Sin embargo, estas salvedades no resuelven el problema de los errores que todavía aparecen en 1674 y que Engling señala.

En tercer lugar, se puede hablar a partir de 1672 de una voluntad y, por consiguiente, también de una decidida intención editorial de Calderón respecto a las comedias de la Cuarta parte. De esta forma, la gestión de la marca editorial para controlar o al menos lucrarse de los beneficios del producto mediante la autentificación de los títulos y la garantía de calidad de los textos, constituye la mejor prueba de lo que llamaremos «intención editorial» de Calderón. Determinar esta intención editorial debería ser el eje de nuestra labor de editores modernos. Esta intención editorial no tiene que ver con los deseos de perfección literaria del autor ni con la posibilidad de interpretar una obra literaria a partir de la supuesta intención del autor, como señalaron Roland Barthes y la crítica posmodernista, sino con la determinación material del recorrido y las variaciones de un cuerpo de información literaria. Este conjunto de información cambia de dos maneras principales, en pequeñas dosis, es decir, en la forma de lo que llamamos errores y variantes, $y$ en pocas pero grandes mutaciones, siempre ligadas a modificaciones en la materialidad del soporte informativo, es decir, cuando se trasladan manuscritos al papel y a la representación y, en segundo lugar, cuando se trasladan impresos al papel, y en concreto, en nuestro caso, al ejecutar la intención editorial de Calderón: cuando Calderón decide que quiere controlar la impresión de sus comedias mediante la fijación de sus textos publicados. De nuevo, el de $E l$ conde Lucanor es significativo:

la calidad textual de la editio princeps es descuidada. Los errores responden a cortes $\mathrm{u}$ omisiones de palabras que dejan trunco el verso o lecturas corruptas de todo tipo: confusión de grafias [...], repetición de palabras [...] $\mathrm{y}$ al intento de subsanar lecciones [...] que no entendió el cajista y que, por supuesto, no hacen sentido alguno ${ }^{21}$.

En cuarto lugar, hemos de evaluar el papel determinante que tiene Vera Tassis respecto a esta Cuarta parte en la consolidación de una determinada forma de la transmisión textual a partir de su edición de 1688. El papel de Vera Tassis como editor de Calderón ha sido estu-

21 Manjarrez, 2007, p. 761. 
diado desde diversos puntos de vista, los cuales se pueden resumir en tres asuntos principales: 1) Su libertad a la hora de intervenir en el texto que, según Hesse, se puede contabilizar como un 52\% de cambios arbitrarios en el conjunto de la Primera a la Cuarta partes ${ }^{22}$. En el caso concreto de El golfo de las sirenas, Vera Tassis concuerda sistemáticamente con las enmiendas hechas por 1674 a 1672, opta por las lecturas de la princeps cuando en 1674 hay un error evidente (lo que demuestra que tuvo acceso a las dos ediciones), pero así y todo se deja llevar por su deseo de mejorar estilísticamente el texto en 145 ocasiones. 2) Una vez que constatamos estas intervenciones estilísticas de Vera Tassis, queda la opción de rechazar la superioridad de la edición de Vera Tassis, como hizo Cotarelo, y como también hace Engling para el caso de La aurora basándose tanto en los errores que aquél introduce en su edición de 1688 respecto a 1672 y 1674, como en sus propias intervenciones, lo que le llevan a concluir que «the Vera Tassis Quarta parte is also misleading and finally corrupt. [...]. This need for a definitive edition of the poet's works becomes pressing when the critical editor appreciates the extent to which the Vera Tassis edition inspired progressive deterioration of the text of $\mathrm{La}_{\text {aurora }}{ }^{23}$. O 3), aceptar la opinión de críticos que, como Cruickshank, destacan sus cualidades editoriales y señalan la modernidad - a pesar de sus excesos- de su labor editorial ${ }^{24}$ y el hecho de que, en comedias como El golfo de las sirenas o el mismo Conde Lucanor, sus lecturas y acotaciones aclaran siempre la situación escénica a la que hace referencia el texto dramático. Como ha señalado Manjarrez: «Lo importante de esta cuestión es tratar de esclarecer el carácter de los "retoques" hechos a los textos, es decir, quedan por determinar las fuentes que emplea el editor para corregir cada una de las comedias de Calderón» ${ }^{25}$. Estudios recientes de obras particulares han demostrado o bien que la calidad de los textos editados por Vera Tassis supera cualquier otro testimonio de la tradición, como el caso de Guárdate del agua mansa ${ }^{26}$, o bien que el editor tuvo acceso o consultó testimonios varios para la prepara-

22 Hesse, 1948, p. 50.

23 Engling, 1994, pp. 42-43.

24 Cruickshank, 2000, p. 35.

25 Cruickshank, 2000, p. 763.

26 Arellano, 1989, p. 71. 
ción de sus ediciones, como en La dama duende ${ }^{27}$ o El mayor monstruo del mundo ${ }^{28}$. Al respecto, Arellano apunta: "cada comedia es un caso distinto y la utilidad de los textos de Vera Tassis es variable y debe ser estudiada en cada ocasión ${ }^{29}$.

En quinto lugar, se puede afirmar que el núcleo de versiones que nos ofrece el paréntesis 1672-1674-1688 se presenta como el foco principal de atención del editor moderno en lo que se refiere a la intención editorial de Calderón. Esta intención editorial vendría determinada no por la superioridad de una de las ediciones, sino por las características que determinan la vida editorial de los textos calderonianos en su desarrollo desde 1672 hasta 1688, es decir:

Expresión de la voluntad editorial de Calderón en el prólogo de 1672. Control de la lista de obras atribuidas.

Correcciones de los errores de la edición de 1672 en 1674 por el propio Calderón y comisión de nuevos errores.

Intervención de Vera Tassis en 1688, a quien en cierta manera se le atribuye una condición especial en cuanto editor de Calderón, para subsanar muchos de esos errores a la vista de los textos de 1672 y 1674, introducción de algunos nuevos errores por su parte, numerosas intervenciones estilísticas y acotaciones clarificadoras de la situación escénica.

¿Qué conclusiones podemos sacar, entonces, en cuanto editores modernos, de la noción de intención editorial de Calderón para las comedias de la Cuarta parte?

Respecto a cómo editar. Editar a partir de concepto de parte y no del de comedia individual, y esto con independencia de cuál sea el formato moderno que se elija para la edición de las comedias. Esto posibilita comenzar el trabajo de edición de las comedias a partir de una muestra relativamente pequeña y accesible de testimonios, lo que acelerará el desarrollo total de la labor.

Respecto a qué editar. En aras de una calculada economía de esfuerzos y de una eficacia filológica basada en la intención editorial del autor, en lo que se refiere a las comedias de esta Cuarta parte, editar a partir de la edición de 1674, cotejando ésta versión con 1672 y con

27 Antonucci, 2000, pp. 109-111.

28 Caamaño Rojo, 2000, pp. 79-90.

29 Arellano, 1989, p. 69. 
la de Vera Tassis de 1688. Por ejemplo, en el caso de El golfo de las sirenas, las variantes indican que la edición de 1674 desciende directamente de la princeps. Calderón remedia la relativa calidad de esta primera edición, revisa y corrige las erratas evidentes en la de 1674, pero aún así acumula otros tantos errores (proceso similar ocurre con El conde Lucanor). A este respecto hay que aclarar que no se puede hablar de dos tiradas diferentes para la edición de 1674. Mediante el cotejo de ambas "tiradas»" se ha comprobado que éstas coinciden exactamente desde los aspectos formales (se trata de una misma composición de imprenta), hasta cuestiones que atañen al ámbito de la ecdótica: corrección de erratas evidentes al texto de la princeps o introducción de nuevas lecturas o errores.

Respecto a las ediciones posteriores a 1688. Tratar estos testimonios, ya sean sueltas o de colecciones, mediante el método del muestreo selectivo. En gran medida, las variantes que presentan todas estas colecciones se han mostrado hasta ahora como irrelevantes, ya que no aproximan el texto calderoniano ni al supuesto arquetipo ni a los manuscritos de la versiones escénicas. Por lo tanto, lo más económico es seleccionar los pasajes más relevantes para la transmisión textual y cotejarlos con las versiones que de ellos ofrecen 1672, 1674 y Vera Tassis.

${ }^{30}$ A propósito de El conde Lucanor, Vitse y Montarnal (1968, p. 56) argumentaban que C presentaba 22 correcciones con respecto a A (1672), también que exhibía menos lecturas erróneas con respecto a $\mathrm{B}$ y «podría, por consecuencia, servir de texto base». B: QUARTA PARTE DE / COMEDIAS / DE DON PEDRO / Calderón de la Barca, Cava / llero del Orden de Santiago. / LLEVA UN PROLOGO DEL / Autor en que distingue las Comedias, que son / Verdaderamente suyas, u no. / ENMEDADAS Y CORREGIDAS EN / esta segunda impresión. / Año [una cruz de Santiago] 1674. CON PRIVELEGIO. En Madrid. Por Bernardo de Hervada. / A costa de Juan de Calatayud Montenegro, Criado y Librero / del Rey nuestro señor. Vendese en su casa en la Plazuela de Santo domingo. Y en Palacio. C: QUARTA PARTE DE / COMEDIAS / DE DON PEDRO / CALDERÓN / DE LA BARCA, CABALLERO / DEL ORDEN DE SANTIAGO. / LLEVA UN PROLOGO DEL AUTOR / en que distingue las Comedias, que son verdadra / mente suyas, u no. / ENMEDADAS, Y CORREGIDAS EN / esta segunda impresión. / Año [una cruz de Santiago] 1674. / CON PRIVELEGIO. En Madrid. Por Bernardo de Hervada. / A costa de Antonio de la Fuente, Mercader de Libros. Ven / dese en su casa enfrente de San Felipe. / Y en palacio. Las copias de los ejemplares corresponden, respectivamente, a los custodiados en la Biblioteca Nacional de Madrid (R30982) para Antonio de la Fuente, y University Library, Cambridge (Hisp.5.68.13) para Juan de Calatayud Montenegro. 
Asimismo, la versión que de estos pasajes den los manuscritos, en caso de que los haya, también es importante y todo ello sin perjuicio del principio establecido por Moll y asumido por Cruickshank: «Cualquier editor que pretenda realizar una labor seria debe consultar el mayor número posible de ejemplares de una edición» ${ }^{31}$.

Desde este punto de vista y teniendo en cuenta que en pocos casos podemos demostrar que los manuscritos sean autógrafos (o que respondan a la intención «pura» de Calderón — más bien responderían a su intención escénica-) y en la mayoría de las casos son filológicamente de menor calidad que los "veratassis» ${ }^{32}$, habrá que concluir que el valor mayor de aquellos reside en la información que suministran respecto a la representación escénica (si la hay) y, sobre todo, respecto a la transmisión textual (¿qué textos podrían haber tenido delante los editores de 1672, 1674 y 1688?). Además, si también tenemos a la vista el llamado proceso de socialización que tiene lugar alrededor de estas tres fechas, hemos de trabajar conjugando dos ideas: una, nuestro criterio actual de calidad filológica (teniendo en cuenta que se trata de textos dramáticos y que una de las características de los textos de Vera es la introducción de acotaciones en este sentido), que parece inclinarnos en muchas ocasiones hacia el trabajo de Vera Tassis, y dos, que parece claro a tenor de la intervención del propio Calderón en los preliminares de esta Cuarta parte que de lo que se trata aquí es de determinar la intención no autorial, sino editorial de Calderón.

Hay que tener en cuenta un esquema horizontal de la transmisión textual de las comedias de esta Cuarta parte, en el que el núcleo formado por las ediciones de 1674, 1672 y Vera Tassis (1688) ocupen un lugar central y el factor tiempo quede representado de izquierda a derecha, y no de arriba abajo, y que divida las ediciones según los criterios de: intención editorial de Calderón; manuscritos e impresiones

31 Cruickshank, 2000, p. 140.

32 Lo expresa en estos términos Germán Vega: «Porque no cabe duda de que la imprenta fue el factor principal de empresa, como las cifras reflejan con total persuasión: de las 122 comedias que con autoría exclusiva o en colaboración hoy podemos leer, sólo dos han sido recuperadas únicamente en manuscritos; por el contrario, suman hasta 37 las que cuentan con impresos como únicos testimonios para elaborar las ediciones críticas, y las 14 que los tienen posteriores a 1700, con escasa posibilidades de que contengan lecturas pertinentes» (Vega García-Luengos, 2002, pp. 15-16). 
anteriores a 1672, con especial atención a la intención de escenificación; colecciones posteriores a 1688; ediciones individuales del siglo xx. De esta forma, quedará más clara la centralidad del trabajo de Vera Tassis en la organización de la historia del texto y quedará difuminado el factor de distorsión que supone la asociación de la precedencia cronológica con la superioridad filológica del texto (que es tan importante en otros casos). Estamos editando una tradición híbrida, en la que es fundamental - y así debe hacer el editor moderno- reflejar la intención editorial del autor, es decir, la transición texto escénicotexto impreso (y vuelta). En nuestro caso, el punto de partida siempre es la intervención de Calderón, aunque a partir de aquí hay que trabajar siempre en dos direcciones: hacia los manuscritos y hacia las ediciones más modernas.

Por último, este esquema basado en los testimonios que nos permiten trabajar sobre la intención editorial de Calderón debe complementarse con los manuscritos y con las ediciones anteriores a 1672 (como es el caso de Fineza contra fineza) en aquellos casos en que existan. Guy Rossetti decide usar la edición de El postrer duelo de España de Bernardo de Hervada de 1674, "as the basic text, abandoning it in favor of Ms. 15273 when it is in obvious error or when the reading of the Ms. is better» ${ }^{33}$. Además de la edición citada, este cotejo es fundamental en El conde Lucanor por la existencia de dos versiones, una atribuida a Calderón y contenida en la Parte quince (1651). En el caso de Apolo y Climene, que cuenta con un manuscrito de 1672 en posesión de la Biblioteca Nacional de Madrid (Mss 16803) y otro de fecha posterior conservado en la Biblioteca Municipal de Madrid (SD $843)^{34}$. Sin embargo, si descontamos la parte de tradición manuscrita, parece que todos los «stemmas» de las comedias incluidas en la Cuarta parte serían iguales, excepto precisamente por esa parte manuscrita que se incluiría en la sección previa a 1672. Ahora bien, la conclusión inicial parece ser que, excepto en casos en los que esos manuscritos puedan ofrecer algo de luz en términos de puesta en escena (como el caso de Fineza contra fineza, dado que se trata de un encargo para una representación), estos manuscritos no ofrecen «nada» para la edición moderna.

33 Rossetti, 1979, p. 82.

${ }^{34}$ Elaine McIlwraith, «Estudio textual de Apolo y Climene». Trabajo inédito. 


\section{Bibliografía}

Antonucci, F., «Contribución al estudio de la historia textual de La dama duende», Criticón, 78, 2000, pp. 109-111.

Arellano, I., "El agua mansa y Guárdate del agua mansa: situación textual», en El agua mansa y Guárdate del agua mansa de Pedro Calderón de la Barca, ed. I. Arellano y V. García, Kassel / Murcia, Reichenberger / Universidad de Murcia, 1989.

Calderón de la Barca, P., Quarta parte (1672), en The Comedias of Calderón. A facsimile Edition Prepared by D. W. Cruickshank \& J. E. Varey with Textual $\&$ Critical Studies, London, Gregg y Tamesis, 1973, vol. X.

- Comedias, Madrid, Biblioteca Castro, 2006, vol. I.

CaAmaño Rojo, M., "El mayor monstruo del mundo» de Calderón de la Barca. Estudio textual, Santiago de Compostela, Universidad de Santiago de Compostela, 2000, pp.79-90.

Cruickshank, D., «The Textual Criticism of Calderón's Comedias: a Survey», en D. W. Cruickshank y J. E. Varey, The Comedias of Calderón. A facsimile edition prepared by D.W. Cruickshank \& J. E. Varey with textual \& critical studies, London, Greg y Tamesis, 1973, vol. I, pp. 1-53.

— "Los "hurtos de la prensa" en las obras dramáticas», en F. Rico (dir.) Imprenta y crítica textual en el Siglo de Oro, estudios al cuidado de P. Andrés y S. Garza, Valladolid, Universidad de Valladolid, 2000, pp. 129-150.

— «Los textos de Calderón», Ínsula, 644-645, 2000, pp. 33-35.

Engling, E. S. «Introduction. Bibliography and the Text», en Pedro Calderón de la Barca, La aurora en Copacabana. Edited with introduction and notes by Ezra S. Engling, London, Tamesis, 1994, pp. 17-47.

Hesse, E.. «The Publication of Calderón's Plays in the Seventeenth Century», Philological Quaterly, 27, 1948, pp. 37-51.

- "The First and the Second Editions of Calderón's Cuarta parte», Hispanic Review, 16, 1948, pp. 209-237.

Manjarrez, G., «La transmisión textual de El conde Lucanor de Calderón de la Barca", en Injerto peregrino de bienes y grandezas admirables. Estudios de literatura y cultura española e hispanoamericana (siglos XVI al XVIII), ed. L. von der Walde, M. J. Rodilla, A. Mejía, G. Illades, A. Higashi y S. González, México, Universidad Autónoma Metropolitana, 2007, pp. 753-767.

Paterson, A. K. G., «La socialización de los textos de Calderón: el legado de don Juan de Vera Tassis y don Pedro de Pando y Mier», en Calderón: innovación y legado. Actas selectas del IX Congreso de la Asociación Internacional de Teatro Español y Novohispano de los Siglos de Oro, en colaboración con el Grupo Investigación Siglo de Oro de la Universidad de Navarra (Pamplona 27 al 29 de marzo de 2000), ed. I. Arellano y G. Vega García-Luengos, New York, Peter Lang Peter, 2001, pp. 17-29. 
Rossetti, G., «Introduction», en Pedro Calderón de la Barca, El postrer duelo de España. Edited with Introduction and Notes by Guy Rossetti, London, Tamesis, 1979, pp. 3-87.

SuÁrez, J. L., "La historización de un mito. El tiempo como problema y solución en Las mocedades del Cid», Revista Canadiense de Estudios Hispánicos 26, 3, 2002, pp. 493-509.

Vega García-Luengos, G., «El predominio de Calderón también en las librerías: consideraciones sobre la difusión impresa de sus comedias», en Calderón 1600-2000. Jornadas de investigación calderoniana, ed. A. González, México, El Colegio de México, 2002, pp. 15-33.

Vitse, M. y L. Montarnal, «Para una edición de El conde Lucanor, de Calderón de la Barca», Segismundo, 7, 1968, pp. 52-72. 